Check for updates

Cite this: RSC Adv., 2020, 10, 28164

Received 3rd March 2020

Accepted 11th July 2020

DOI: 10.1039/d0ra02031a

rsc.li/rsc-advances

\section{Fluorescent polydopamine nanoparticles as a nanosensor for the sequential detection of mercury ions and L-ascorbic acid based on a coordination effect and redox reaction $\uparrow$}

\author{
Yi-Xuan Yang, \$Yan-Zhao Fang, $\$$ Jing-Xuan Tian, Qiang Xiao (DD * \\ and Xiang-Juan Kong (D)
}

\begin{abstract}
Herein, a novel fluorescence nanosensor using intrinsic fluorescent polydopamine nanoparticles (PDA NPs) as an effective signal reporter has been constructed for the simple, rapid and sequential detection of mercury ions $\left(\mathrm{Hg}^{2+}\right)$ and L-ascorbic acid (AA) based on a coordination effect and redox reaction. The fluorescence of the PDA NPs could be specifically quenched by $\mathrm{Hg}^{2+}$ through intense coordination effects between the $\mathrm{Hg}^{2+}$ and the groups (catechol, amine, ketone and imine) on the surface of the PDA NPs. However, when AA and $\mathrm{Hg}^{2+}$ coexisted in solution, the fluorescence of the PDA NPs pronouncedly recovered via the redox reaction of $\mathrm{Hg}^{2+}$, with it being reduced to $\mathrm{Hg}^{0}$ by $\mathrm{AA}$. The fluorescence quenching mechanism of $\mathrm{Hg}^{2+}$ towards the PDA NPs and the redox reaction between $\mathrm{Hg}^{2+}$ and AA were also fully investigated. The nanosensor exhibited high sensitivity and desirable selectivity for $\mathrm{Hg}^{2+}$ and $\mathrm{AA}$ detection. Moreover, the strategy was successfully explored in real samples (tap water, lake water and human serum samples) with satisfactory recoveries. The developed nanosensor provides new sights and good inspiration for $\mathrm{Hg}^{2+}$ and $\mathrm{AA}$ detection under real conditions.
\end{abstract}

\section{Introduction}

Mercury ions $\left(\mathrm{Hg}^{2+}\right)$, as a highly toxic heavy metal pollutant that abound in the environment, may lead to serious side effects once they enter living organisms, because they are not biodegradable and can accumulate in the body, leading to kidney, eye, respiratory and central nervous system damage. ${ }^{\mathbf{1 , 2}}$ L-Ascorbic acid (AA), an essential micronutrient and water-soluble antioxidant, plays a vital role in numerous physiological and biochemical systems, such as oxidative stress reduction, immune cell function and disease prevention in the human body. ${ }^{3,4}$ Moreover, abnormal levels of AA in the human body are closely associated with many diseases, such as rheumatoid arthritis, scurvy, kidney stones, and diarrhea. ${ }^{5-9}$ Therefore, developing simple, rapid, and economical methods for the highly sensitive and selective detection of $\mathrm{Hg}^{2+}$ and $\mathrm{AA}$ has attracted considerable interest owing to their significant impact on human health.

Up to now, a number of strategies based on electrochemistry, ${ }^{10,11}$ chemiluminescence, ${ }^{\mathbf{1 2 , 1 3}}$ chromatography, ${ }^{\mathbf{1 4 , 1 5}}$

Jiangxi Key Laboratory of Organic Chemistry, Jiangxi Science and Technology Normal University, Nanchang 330013, P. R. China. E-mail: xiaoqiang@tsinghua.org.cn; xiangjuankong@163.com; Fax: +86-791-86422903; Tel: +86-791-86422903

$\dagger$ Electronic supplementary information (ESI) available: Experimental details and additional information. See DOI: 10.1039/d0ra02031a

\$ These authors contributed equally to this work. colorimetry, ${ }^{\mathbf{1 6}, \mathbf{1 7}}$ fluorescence methods and other techniques have been introduced for $\mathrm{Hg}^{2+}$ and AA detection. ${ }^{18-23}$ Among them, fluorescence methods have been widely used due to their high sensitivity, rapid response and good reproducibility. ${ }^{24,25}$ Up to now, nanoparticles, including upconversion nanoparticles, ${ }^{7,26-28}$ have emerged for $\mathrm{Hg}^{2+}$ and AA detection. ${ }^{29-34}$ Nevertheless, the preparation procedures and reaction conditions of these nanoparticles might be sophisticated and harsh. Thus, developing simple and rapid fluorescence strategies for $\mathrm{Hg}^{2+}$ and AA detection is still highly desirable.

More recently, intrinsically fluorescent polydopamine nanoparticles (PDA NPs) have emerged as a novel bioinspired polymer nanoprobe owing to their easy synthesis, good watersolubility and biocompatibility. ${ }^{35,36}$ To the best of our knowledge, intrinsically fluorescent PDA NPs have emerged as a burgeoning field with brilliant prospects in sensing and imaging applications. For instance, Liu et al. developed a fluorescent polydopamine dot-based nanosensing strategy for glutamate and $\mathrm{Al}^{3+}$ detection in human serum and living cells. $^{37} \mathrm{Fu}$ et al. reported a facile one-pot method for the synthesis of blue luminescent PDA NPs and developed a nanosystem for the sensitive detection of $\mathrm{Fe}^{3+} \cdot{ }^{38}$ Chu et al., using $\mathrm{MnO}_{2}$ as an oxidant, synthesized the intrinsically fluorescent PDA NPs and established a nanosensor for glutathione detection. $^{39}$ Yang et al. extended the application of the intrinsically fluorescent PDA NPs towards the sensitive 


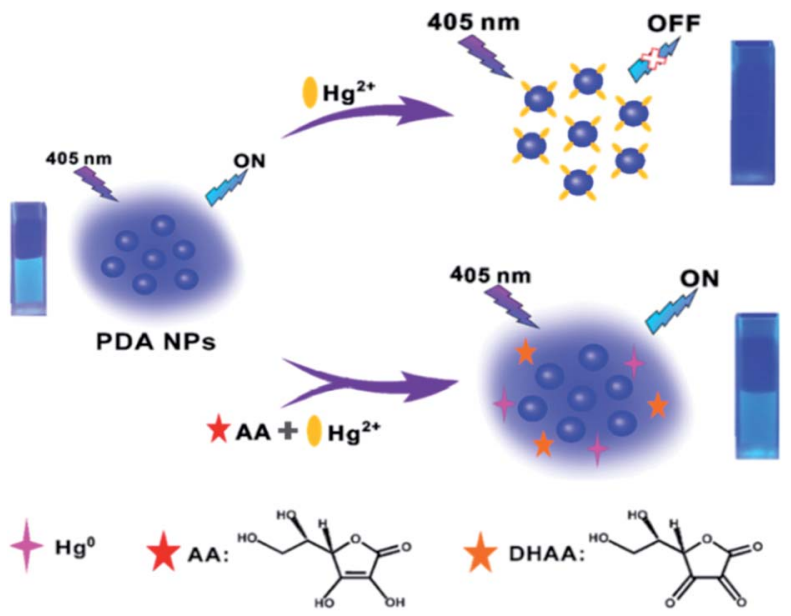

Scheme 1 Schematic illustration of the mechanism of the fluorescent PDA NPs-based nanosensor for $\mathrm{Hg}^{2+}$ and AA detection.

detection of alkaline phosphatase by employing $\mathrm{MnO}_{2}$ nanosheets as an effective nanoquencher. ${ }^{40}$ Jiang et al., using dopamine as a precursor, successfully synthesized fluorescent polydopamine organic nanoparticles, which were further used for developing nucleus-targeting imaging platforms. ${ }^{41}$ The intrinsically fluorescent PDA NPs presented outstanding physicochemical features and displayed promising applications for sensing and imaging. However, the exploitation of intrinsically fluorescent PDA NPs for analytical applications is still at its early stages, and constructing more novel designs and exploring groundbreaking systems for sensing applications is still attractive.

Herein, we, for the first time, propose a novel fluorescence nanosensor for the sequential detection of $\mathrm{Hg}^{2+}$ and AA based on $\mathrm{Hg}^{2+}$-induced quenching of intrinsically fluorescent PDA NPs through an intense coordination effect between $\mathrm{Hg}^{2+}$ and the groups on the surface of the PDA NPs, and AA-triggered pronounced fluorescence recovery of PDA NPs via redox reaction of $\mathrm{Hg}^{2+}$, with them being reduced to $\mathrm{Hg}^{0}$ by AA. As shown in Scheme 1, the fluorescence of the PDA NPs showed a selective quenching response towards $\mathrm{Hg}^{2+}$ via a coordination effect. Interestingly enough, when $\mathrm{AA}$ and $\mathrm{Hg}^{2+}$ were added simultaneously into the solution, $\mathrm{Hg}^{2+}$ was quickly reduced to $\mathrm{Hg}^{0}$, meanwhile, AA, acting as a reductant, was oxidized to dehydroascorbic acid (DHAA), ${ }^{42}$ as shown in the following equation:

$$
\mathrm{Hg}^{2+}+\mathrm{AA} \rightarrow \mathrm{Hg}^{0}+\mathrm{DHAA}+2 \mathrm{H}^{+}
$$

And then, as the concentration of AA increased, the fluorescence intensity of the PDA NPs enhanced progressively because of the elimination of the quenching effect. The fluorescent PDA NPs-based nanosensor displayed high sensitivity and selectivity to $\mathrm{Hg}^{2+}$ and AA assay. Moreover, the proposed sensor displayed excellent analytical potential for $\mathrm{Hg}^{2+}$ and AA detection in real samples, demonstrating promising applications in environmental analysis and biological detection research.

\section{Experimental}

\section{Reagents and materials}

Reduced $N$-ethylmaleimide (NEM) and dopamine hydrochloride were purchased from Sigma-Aldrich. Glutathione (GSH), leucine (Leu), glycine (Gly), cysteine (Cys), homocysteine (Hcy), and AA were purchased from Sangon Biotechnology Company. All the other chemical reagents were of analytical grade. The water used throughout the experiments was obtained from a water purification system with an electric resistance of $>18.2$ $\mathrm{M} \Omega$ (Sartorius, Germany). Tap water was obtained from our lab and lake water was obtained from Jingguan Lake at our school. Human serum samples were provided by The First Hospital of Nanchang, Nanchang University (Nanchang, China). All animal procedures were performed in accordance with the Guidelines for the Care and Use of Laboratory Animals of The First Hospital of Nanchang, Nanchang University and approved by the Animal Ethics Committee of The First Hospital of Nanchang, Nanchang University. Our institutional ethics committee specifically approved the absence of informed consent because the data were analyzed anonymously.

Fluorescence measurements were performed in a 96-well black microplate on a Varioskan LUX microplate reader under $405 \mathrm{~nm}$ excitation light (Thermo Scientific, USA). The accurate experimental spectroscopic procedure was carried out at a bandwidth of $12 \mathrm{~nm}$. UV-vis absorption measurements were performed in an 8453 UV-vis spectrophotometer (Agilent, USA). Fourier-transform infrared (FT-IR) spectra were measured using a Spectrum Two spectrometer (PerkinElmer, USA). Transmission electron microscopy (TEM) and energy-dispersive X-ray spectroscopy (EDS) images were acquired using a TECNAI G ${ }^{2} 20$ at an accelerating voltage of $200 \mathrm{kV}$. X-ray photoelectron spectroscopy (XPS) spectra of the solid samples were measured on an Axis Ultra DLD spectrometer (Shimadzu, Japan).

\section{Synthesis of fluorescent PDA NPs}

The synthesis of fluorescent PDA NPs was carried out according to the reported methods with slight modification. ${ }^{31,43}$ Firstly, the fluorescent PDA NPs were formed by oxidative polymerization via the addition of an aqueous solution of dopamine hydrochloride $(40 \mathrm{mM}, 200 \mu \mathrm{L})$ and sodium hydroxide solution $(100 \mathrm{mM}, 320 \mu \mathrm{L})$ into phosphate buffer (PB) $(2 \mathrm{mM}, \mathrm{pH}$ 7.4, $7.08 \mathrm{~mL}$ ) under magnetic stirring for $1 \mathrm{~h}$ at room temperature. Thereafter, hydrochloric acid $(0.2 \mathrm{M}, 400 \mu \mathrm{L})$ was introduced with stirring for an additional $30 \mathrm{~min}$ to sharply reduce the polymerization speed. For purification, the prepared PDA NPs solution was centrifuged for 30 minutes at $12000 \mathrm{rpm}$ to remove large nonfluorescent PDA NPs. The obtained supernatant was stored at $4{ }^{\circ} \mathrm{C}$ for future use.

\section{$\mathrm{Hg}^{2+}$ and AA detection in aqueous solution and real samples}

For $\mathrm{Hg}^{2+}$ detection, varying concentrations of $\mathrm{Hg}^{2+}$ were added into the solution containing $50 \mu \mathrm{L}$ of $\mathrm{PB}(\mathrm{pH} 7.0,2 \mathrm{mM})$ solution and $10 \mu \mathrm{L}$ as-prepared fluorescent PDA NPs, and enough sterilized water was added into the mixture to adjust the final volume to $100 \mu \mathrm{L}$. After mixing, the reaction proceeded at room 
temperature for $30 \mathrm{~min}$. And then, the solution was subjected to fluorescence measurements.

For AA detection, varying concentrations of AA and $20 \mu \mathrm{M}$ $\mathrm{Hg}^{2+}$ were added into the solution containing $50 \mu \mathrm{L}$ of $\mathrm{PB}(\mathrm{pH}$ 7.0, $2 \mathrm{mM}$ ) solution and $10 \mu \mathrm{L}$ of the as-prepared fluorescent PDA NPs, and enough sterilized water was added into the mixture to adjust the final volume to $100 \mu \mathrm{L}$. After mixing, the reaction was allowed to proceed at room temperature for $15 \mathrm{~min}$, and was then subjected to fluorescence measurements.

The practicality of the sensor for $\mathrm{Hg}^{2+}$ detection was demonstrated in tap water, lake water and diluted human serum samples, and for AA detection was demonstrated in diluted human serum samples. A standard addition recovery method was used for evaluating the feasibility of the fluorescent PDA NPs for $\mathrm{Hg}^{2+}$ and AA detection in real samples. In particular, a 20-fold diluted human serum sample was firstly treated with NEM (1.0 mM) and incubated for $30 \mathrm{~min}$ to eliminate any interference from sulfhydryl compounds including Cys, Hcy, GSH and $\mathrm{Na}_{2} \mathrm{~S}$ (using as $\mathrm{H}_{2} \mathrm{~S}$ donor) before testing.

\section{Results and discussion}

\section{Synthesis and characterization of the fluorescent PDA NPs}

The fluorescent PDA NPs were easily synthesized by oxidation and polymerization of dopamine molecules under basic conditions with stirring. The solution was acidified by adding hydrochloric acid to lower the polymerization speed and then fluorescent PDA NPs formed. ${ }^{43}$ Fig. 1A showed that the excitation wavelength of the fluorescent PDA NPs was at around $405 \mathrm{~nm}$ and the emission intensity reached a maximum at around $468 \mathrm{~nm}$. Apparently, Fig. 1B showed that the emission peak of PDA NPs was excitation-independent, and the excitation wavelength at $405 \mathrm{~nm}$ gave the maximum fluorescence output. Fig. S1 $\uparrow$ shows that the fluorescence signal of the PDA NPs has no obvious decrease within seven days and under continuous
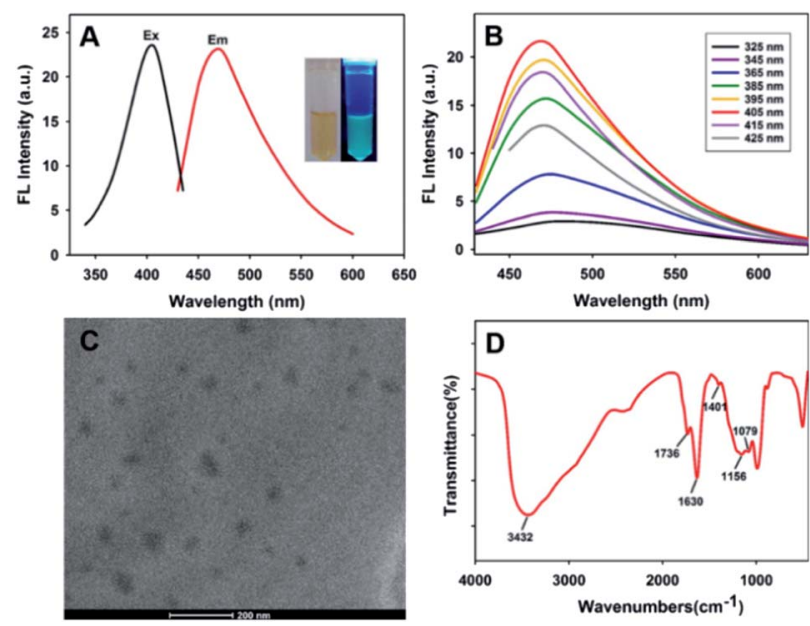

Fig. 1 (A) Excitation and emission spectra of the synthesized PDA NPs. The inset shows photographs of the PDA NPs under room light and $365 \mathrm{~nm}$ UV light irradiation. (B) Fluorescence emission spectra of the PDA NPs under different excitation wavelengths. (C) TEM images of the PDA NPs. (D) FT-IR results. irradiation with $405 \mathrm{~nm}$ light, revealing the outstanding photostability of the formed PDA NPs and the potential for analysis application.

The size and shape of the formed PDA NPs were characterized by TEM. Fig. 1C shows that the PDA NPs were monodispersed and irregular shaped with diameters in the range of 20-60 nm. Compositional analysis of the formed PDA NPs by EDS exhibited the signals of the C, $\mathrm{N}$ and $\mathrm{O}$ elements (Fig. S2 $\dagger$ ). Finally, FT-IR spectroscopy was used to determine the surface chemical groups of the PDA NPs. As shown in Fig. 1D, the strong and broad band located at $3432 \mathrm{~cm}^{-1}$ was attributable to the presence of $\mathrm{N}-\mathrm{H}$ bond and phenolic $\mathrm{O}-\mathrm{H}$ bond stretching vibrations, the peak appeared at $1401 \mathrm{~cm}^{-1}$ corresponded to the bending vibrations of $\mathrm{O}-\mathrm{H}$ bonds, and the peak located at $1630 \mathrm{~cm}^{-1}$ was assigned to the presence of $\mathrm{N}-\mathrm{H}$ bond bending vibrations and $\mathrm{C}=\mathrm{C}$ bond stretching vibrations. The peak at $1736 \mathrm{~cm}^{-1}$ was ascribed to $\mathrm{C}=\mathrm{O}$ bonds, the peak at around $1156 \mathrm{~cm}^{-1}$ corresponded to the stretching vibration of $\mathrm{C}-\mathrm{N}$ bonds, and the peak located at around $1079 \mathrm{~cm}^{-1}$ was attributable to the stretching vibrations of $\mathrm{C}-\mathrm{O}$ bonds. The results proved the existence of a number of ketone and hydrophilic groups (catechol, amine, imine) on the surface of the formed PDA NPs, which not only resulted in their excellent water solubility but also provided a platform for metal ion coordination. The water solubility of PDA NPs in water was calculated to be $18.312 \mathrm{mg} \mathrm{mL}^{-1}$, which was determined by dissolving excess lyophilized PDA NPs in deionized water. The exhibited water solubility makes it an attractive candidate for sensing applications in solution.

\section{Feasibility analysis of the sensing system for $\mathrm{Hg}^{2+}$ and $\mathrm{AA}$ detection}

The feasibility of the proposed strategy was verified using fluorescence and UV-vis absorption spectroscopic measurements. Fig. 2 shows that the fluorescence signal of the PDA NPs decreased dramatically in the presence of $\mathrm{Hg}^{2+}$ (black line). However, the fluorescence signal of the PDA NPs greatly increased upon the addition of AA (green line), which was comparable to the fluorescence intensity of the PDA NPs (red

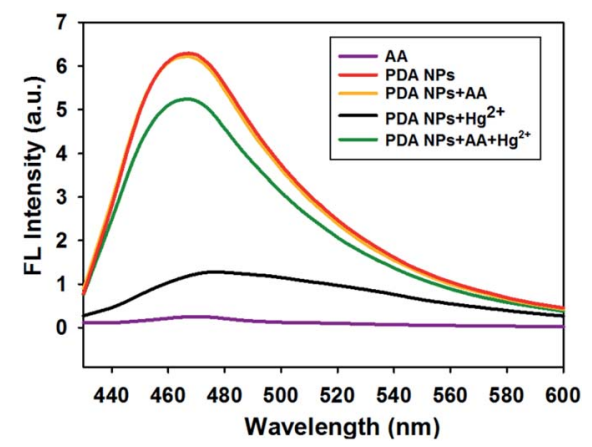

Fig. 2 Fluorescence emission spectral responses obtained under different conditions: AA (purple line); PDA NPs (red line); PDA NPs + AA (orange line); PDA NPs $+\mathrm{Hg}^{2+}$ (black line); $\mathrm{PDANPs}+\mathrm{Hg}^{2+}+\mathrm{AA}$ (green line). 
line) or PDA NPs with AA (orange line), suggesting that $\mathrm{Hg}^{2+}$ effectively quenched the fluorescence of the PDA NPs and AA successfully recovered the fluorescence signal of the system via a redox reaction between the AA and $\mathrm{Hg}^{2+}$. UV-vis absorption spectra of the system were also measured, where Fig. S3† shows that the absorption peak shifted remarkably to $286 \mathrm{~nm}$ in the presence of $\mathrm{Hg}^{2+}$ (blue line). In contrast, when $\mathrm{AA}$ and $\mathrm{Hg}^{2+}$ coexisted in the solution, the absorption peak was observed at $280 \mathrm{~nm}$ (green line), which was the same as the absorption peak of the system without $\mathrm{Hg}^{2+}$ (red line). Furthermore, zeta potential measurements revealed the changes in the surface charge of the PDA NPs. Fig. S4 $\uparrow$ shows that the coordination of $\mathrm{Hg}^{2+}$ significantly shifted the zeta potential from -46.17 to $-33.63 \mathrm{mV}$, and after AA was added to the system, the zeta potential shifted to $-41.23 \mathrm{mV}$ again. The above results gave clear evidence for the feasibility of the system.

\section{Mechanism of $\mathrm{Hg}^{2+}$-induced fluorescence quenching toward the PDA NPs}

The fluorescence quenching process occurs primarily via static and dynamic modes. The static mode involves the formation of a nonfluorescent complex via a reaction between a fluorescence molecule in the ground state and a quencher, accompanied by fluorescence intensity decreases but the excited-state fluorescence lifetime of the fluorophore does not change. On the contrary, the dynamic mode involves a collision between the excited fluorescence molecule and the quencher, which leads to an excited-state fluorescence lifetime change of the fluorophore. ${ }^{44-47}$ The $\mathrm{Hg}^{2+}$-induced fluorescence quenching mechanism toward the PDA NPs was further investigated via fluorescence decay curves. Fig. 3 shows that the fluorescence lifetime of PDA NPs in the absence of $\mathrm{Hg}^{2+}$ fitted well with a monoexponential function, and the fluorescence life time of the PDA NPs was calculated to be 4.36 ns. Interestingly, in the presence of $\mathrm{Hg}^{2+}$, the fluorescence lifetime of the PDA NPs was well fitted with a biexponential function, consisting of a short lifetime component of $\tau_{1}=0.75 \mathrm{~ns}(49.17 \%)$ and a long lifetime component of $\tau_{2}=4.49 \mathrm{~ns}(50.83 \%)$. The significantly decreased fluorescence lifetime of $\tau_{1}$ might result from the occurrence of charge transfer from the PDA NPs to $\mathrm{Hg}^{2+}$, which led to the quenching of the fluorescence and proved the existence of

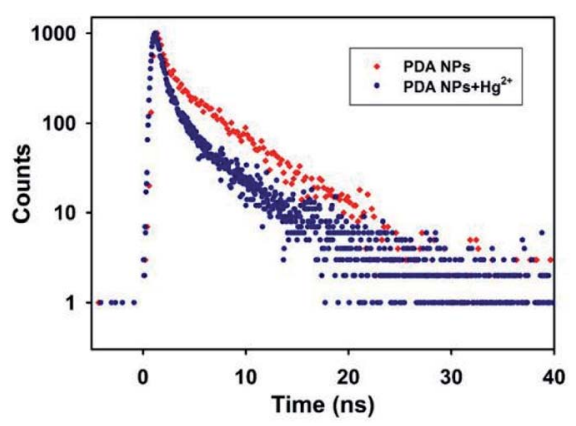

Fig. 3 Fluorescence decay curves of the PDA NPs in the absence and presence of $\mathrm{Hg}^{2+}$ with the emission monitored at $468 \mathrm{~nm}$ and excitation at $375 \mathrm{~nm}$. dynamic quenching. The unaffected fluorescence life time of $\tau_{2}$ might be due to the formation of a non-fluorescent complex between $\mathrm{Hg}^{2+}$ and the PDA NPs, which confirmed the mode of static quenching. The results validated the fluorescence quenching of the PDA NPs by $\mathrm{Hg}^{2+}$ was a combination of static and dynamic quenching processes.

\section{Demonstration of the redox reaction between $\mathrm{AA}$ and $\mathrm{Hg}^{2+}$}

With regard to the demonstration of the redox reaction between the AA and $\mathrm{Hg}^{2+}$, XPS spectral analysis was carried out on the surfaces of the PDA NP samples. Fig. 4A shows the survey spectrum that contains $\mathrm{C} 1 \mathrm{~s}, \mathrm{~N} 1 \mathrm{~s}, \mathrm{O} 1 \mathrm{~s}$, and $\mathrm{Hg}$ if peaks. The high-resolution $\mathrm{Hg}$ if spectrum (Fig. 4B) shows two peaks located at $101.2 \mathrm{eV}$ for $\mathrm{Hg} 4 \mathrm{f}_{7 / 2}$ and $105.2 \mathrm{eV}$ for $\mathrm{Hg} 4 \mathrm{f}_{5 / 2}$. The location of the peaks is characteristic of $\mathrm{Hg}^{2+} .^{48}$ In contrast, the presence of the AA in the system resulted in $\mathrm{Hg}^{2+}$ reduction to $\mathrm{Hg}^{0}$, which was not coordinated by the PDA NPs. When analyzing PDA NPs treated with $\mathrm{Hg}^{2+}$ in the presence of AA, the peaks for $\mathrm{Hg}$ 4f disappeared in both the survey (Fig. 4C) and high-resolution (Fig. 4D) $\mathrm{Hg}$ 4f spectra. The results indicated the occurrence of the redox reaction of $\mathrm{Hg}^{2+}$ being reduced to $\mathrm{Hg}^{0}$ by $\mathrm{AA}$, as was previously reported. ${ }^{49}$

\section{Optimization of the experimental conditions}

Before investigating the detection behavior of the sensing system, several experimental parameters, including the acidity $(\mathrm{pH})$ of the solution and reaction time for $\mathrm{Hg}^{2+}$ detection, and the adding order of the chemicals and reaction time for AA detection were investigated to systematically optimize the experimental conditions of the detection method.

Optimization of the experimental conditions for $\mathbf{H g}^{2+}$ detection. The influence of the solution $\mathrm{pH}$ was first demonstrated because the protonation and deprotonation of the amine, imine, and phenol groups on the surface of the PDA NPs were dramatically changed according to the variation of the
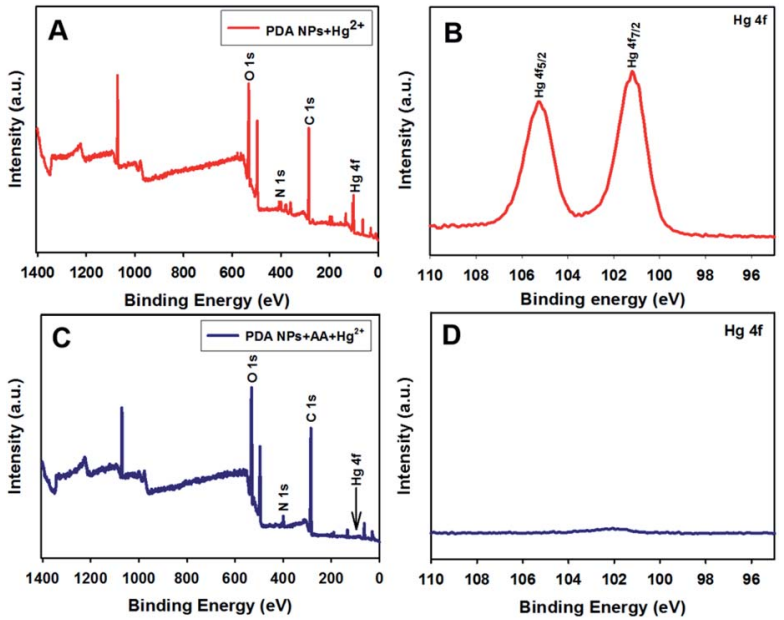

Fig. 4 XPS analysis and high-resolution XPS spectra of $\mathrm{Hg}^{2+}$ on the surface of the PDA NPs in the absence ( $A$ and $B$ ) and presence of AA ( $C$ and D). 
solution $\mathrm{pH}$, thus resulting in different quenching responses of the PDA NPs towards $\mathrm{Hg}^{2+}$. Fig. S5A $\dagger$ shows that the fluorescence intensity increased when the solution $\mathrm{pH}$ was increased from 5.0 to 6.5, and remained stable in the $\mathrm{pH}$ range of 7.0-9.0. However, once $\mathrm{Hg}^{2+}$ was added, the fluorescence intensity decreased obviously in the $\mathrm{pH}$ range of 4.0-7.0, and then gradually increased when the $\mathrm{pH}$ of the solution was increased from 7.5 to 9.0, as shown in Fig. S5B. $\dagger$ Therefore, a solution $\mathrm{pH}$ of 7.0 was selected for further study.

To realize the fast and highly sensitive detection of $\mathrm{Hg}^{2+}$, the reaction time of the system for $\mathrm{Hg}^{2+}$ detection was investigated. Fig. $\mathrm{S} 5 \mathrm{C} \dagger$ shows that the fluorescence intensity of the PDA NPs decreased sharply when the reaction time was varied from 0 to $30 \mathrm{~min}$, and then remained unchanged after $30 \mathrm{~min}$. Thus, 30 min was used for the subsequent experiments.

Optimization of the experimental conditions for AA detection. Most notably, the increase in the fluorescence intensity of the sensing system for AA detection was dependent on order that the chemicals were added into the solution. As shown in Fig. S6A, $\uparrow$ if the PDA NPs and $\mathrm{Hg}^{2+}$ were first added to the solution and it was kept at room temperature for $30 \mathrm{~min}$ the PDA NPs exhibited an inconspicuous fluorescence signal after the addition of the AA even after it was reacted at room temperature for $45 \mathrm{~min}$ (orange line, group A), which might be ascribed to the strong coordination reaction between the $\mathrm{Hg}^{2+}$ and the groups on the surface of the PDA NPs that interfere with the redox reaction between the $\mathrm{AA}$ and $\mathrm{Hg}^{2+}$. By contrast, in group B, the PDA NPs and $\mathrm{Hg}^{2+}$ were first added into the solution followed by the addition of the AA immediately, in which the PDA NPs displayed significant fluorescence signal enhancement (blue line) after $30 \mathrm{~min}$ of co-incubation. In particular, in the group $\mathrm{C}$ adding of the chemicals in the order of PDA NPs, AA and $\mathrm{Hg}^{2+}$, desirable fluorescence signal response (red line) was observed after $30 \mathrm{~min}$ of co-incubation. Hence, to realize highly sensitive AA detection, the addition order of group $\mathrm{C}$ was chosen for further study.

To realize the rapid detection of AA, the reaction time of the system for AA detection was also investigated. Fig. S6B $\uparrow$ shows that the fluorescence intensity of the PDA NPs increased rapidly when the reaction time varied from 0 to $15 \mathrm{~min}$, and then leveled off at $15 \mathrm{~min}$. Thus, $15 \mathrm{~min}$ was used for subsequent experiments.

\section{Detection procedure}

The fluorescence responses of the nanosensor to $\mathrm{Hg}^{2+}$ and $\mathrm{AA}$ in buffer solutions were investigated under the optimal experimental conditions. Fig. 5A shows that the fluorescence intensity of the PDA NPs decreased sharply when the concentration of $\mathrm{Hg}^{2+}$ increased from 0 to $10 \mu \mathrm{M}$, and then became sluggish even when the concentration of $\mathrm{Hg}^{2+}$ was increased beyond $10 \mu \mathrm{M}$. Good linearity between the relative fluorescence intensity and the concentration of $\mathrm{Hg}^{2+}(0-10 \mu \mathrm{M})$ was obtained $\left(R^{2}=0.9801\right)$, as shown in Fig. 5B, and the detection limit was found to be 0.19 $\mu \mathrm{M}$ based on the $3 \sigma$ rule. Fig. $5 \mathrm{C}$ shows the fluorescence spectral responses of the sensor with different concentrations of AA. As expected, the fluorescence intensity correlated well upon
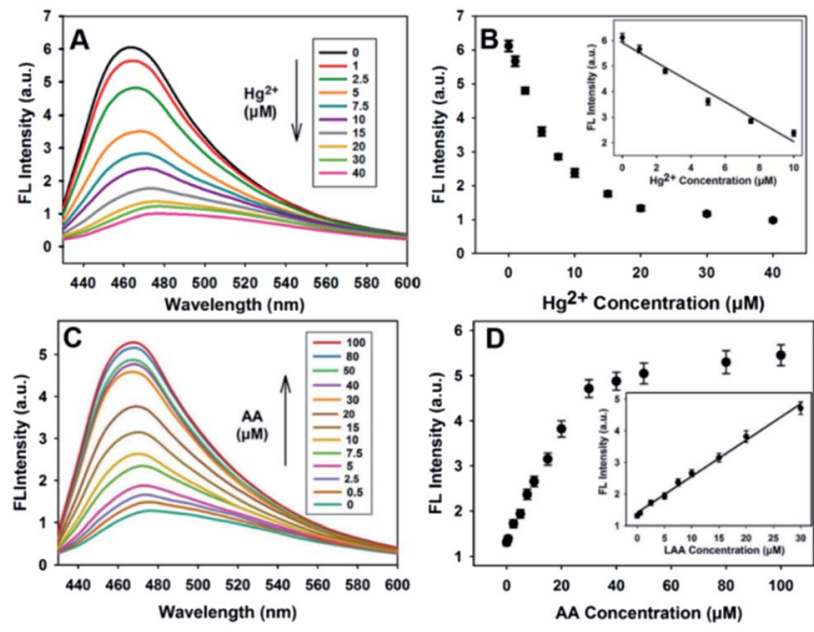

Fig. 5 (A) The fluorescence spectral responses of the PDA NPs towards a series of various $\mathrm{Hg}^{2+}$ concentrations. (B) Relationship between the fluorescence intensity of the PDA NPs at $468 \mathrm{~nm}$ and the $\mathrm{Hg}^{2+}$ concentrations $(0-40 \mu \mathrm{M})$. The inset shows the linear range from 0 to $10 \mu \mathrm{M}$. (C) Fluorescence profiles of the PDA NPs in the presence of various AA concentrations $(0-100 \mu \mathrm{M})$. (D) The corresponding fluorescence spectral responses versus $A A$ concentrations. The inset shows the linear range from 0 to $30 \mu \mathrm{M}$. The error bars represent the standard deviation of three repeated experiments.

increasing the concentration of $\mathrm{AA}(0-100 \mu \mathrm{M})$. The fluorescence intensity apparently increased, which was as a result of the redox reaction between the $\mathrm{AA}$ and $\mathrm{Hg}^{2+}$. Fig. 5D shows that there is good linearity between the relative fluorescence intensity and the concentration of AA up to a concentration of $30 \mu \mathrm{M}$ was reached $\left(R^{2}=0.9934\right)$. The detection limit was found to be $0.4 \mu \mathrm{M}$ based on the $3 \sigma$ rule. The detection results for $\mathrm{Hg}^{2+}$ and AA were better than or comparable to the results in previous reports (Table S1 $\dagger$ ), demonstrating that the developed sensor has many obvious advantages, for instance, simplicity, rapid implementation and high sensitivity for $\mathrm{Hg}^{2+}$ and AA detection.

\section{Selectivity of the proposed sensor}

The selectivity of the proposed method for $\mathrm{Hg}^{2+}$ and AA detection was evaluated in the presence of a variety of possible interferences. Fig. 6A shows that when the fluorescent PDA NPs were incubated with $\mathrm{Hg}^{2+}, \mathrm{Cu}^{2+}$ and $\mathrm{Fe}^{2+}$, the fluorescence intensity diminished compared with the other metal ions. While $\mathrm{Fe}^{2+}$ and $\mathrm{Cu}^{2+}$ ions influence the mercury detection of the PDA NPs, these inputs can in the future be separated using a logic gate concept. ${ }^{50,51}$ Also, Fig. 6B shows that significant fluorescence intensity enhancements were conspicuously observed in the presence of Hcy, Cys, GSH, $\mathrm{NaHSO}_{3}, \mathrm{Na}_{2} \mathrm{~S}$ and AA, while other interfering species showed slight changes, even at higher concentration. Fortunately, the interferences from $\mathrm{Hcy}$, Cys, GSH, $\mathrm{NaHSO}_{3}$ and $\mathrm{Na}_{2} \mathrm{~S}$ could be effectively eliminated by NEM, a specific scavenger that forms stable thioether bonds with sulfhydryl compounds, whereas the fluorescence intensity of the PDA NPs for AA detection was essentially unchanged after the introduction of NEM. Notably, $\mathrm{I}^{-}$caused a slight fluorescence increase when the concentration was 100 

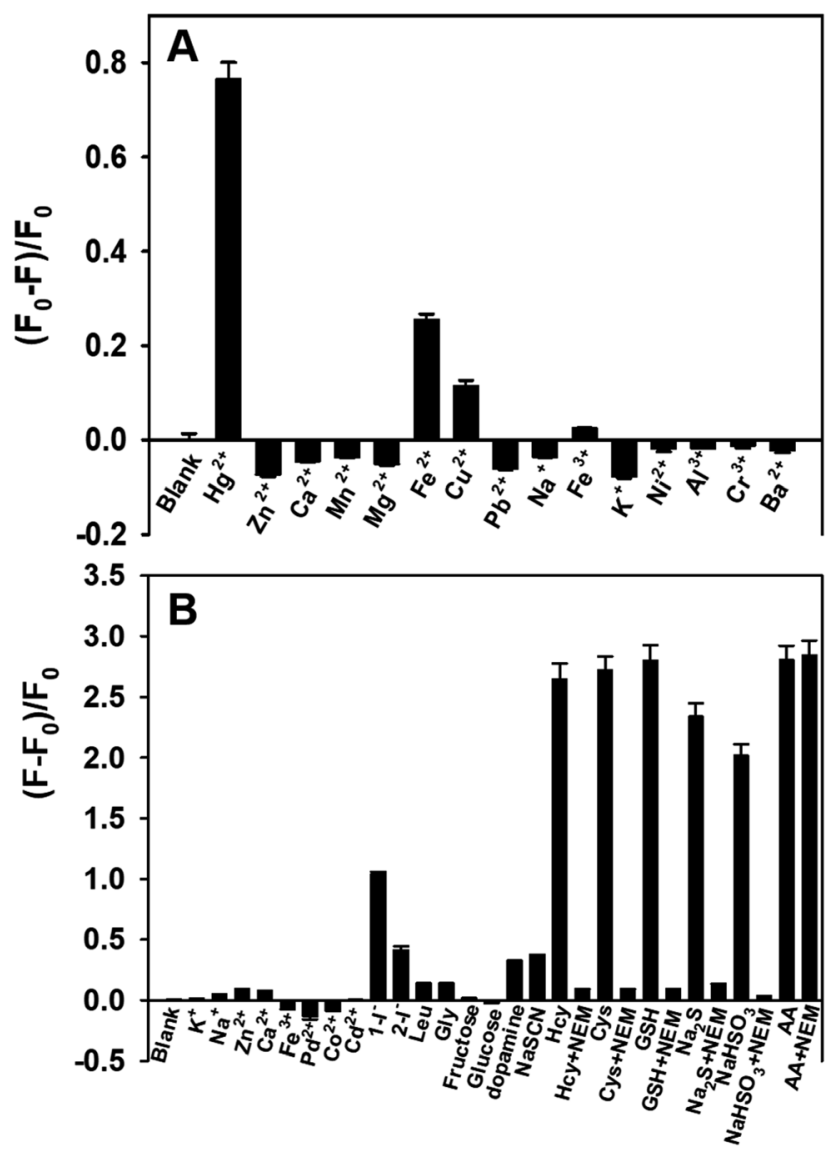

Fig. 6 (A) Selectivity of the sensor for $\mathrm{Hg}^{2+}$ detection. The concentrations of $\mathrm{Hg}^{2+}, \mathrm{Fe}^{2+}, \mathrm{Cu}^{2+}$ and $\mathrm{Al}^{3+}$ were $20 \mu \mathrm{M}$, the concentrations of the other metal ions were $100 \mu \mathrm{M}$. $F_{0}$ and $F$ are the fluorescence intensities of the PDA NPs at $468 \mathrm{~nm}$ in the absence and presence of metal ions, respectively. (B) Selectivity of the sensor for AA detection. The concentrations of $\mathrm{Pd}^{2+}, \mathrm{I}^{-}, \mathrm{AA}$, dopamine, NaSCN$, \mathrm{Hcy}, \mathrm{Cys}, \mathrm{GSH}$ and $\mathrm{Na}_{2} \mathrm{~S}$ were $100 \mu \mathrm{M}$, the concentrations of other interfering species were $1 \mathrm{mM}$ and the NEM concentration was $0.6 \mathrm{mM} .1-I^{-}$represents that $\mathrm{I}^{-}$was added before $\mathrm{Hg}^{2+}, 2-\mathrm{I}^{-}$represents that $\mathrm{I}^{-}$was added after $\mathrm{Hg}^{2+} . F_{0}$ and $F$ are the fluorescence intensities of the PDA NPs in the absence and presence of interfering species or AA, respectively. The error bars represent the standard deviation of three repeated experiments.

$\mu \mathrm{M}$, but it could be minimized by changing order in which the chemicals were added. In addition, the fluorescence signals caused by dopamine and NaSCN were nearly negligible when the concentrations of them were the same as AA. The results fully indicated the high selectivity of the nanosensor for $\mathrm{Hg}^{2+}$ and AA detection.

\section{Analysis of $\mathrm{Hg}^{2+}$ and $\mathrm{AA}$ in real samples}

The practical utility of the nanosensor for $\mathrm{Hg}^{2+}$ and AA detection in complex media was demonstrated in real samples (tap water, lake water and diluted human serum samples). With four different concentrations of $\mathrm{Hg}^{2+}$ spiked into tap water, lake water and the diluted human serum samples respectively, recoveries of between $93.4 \%$ and $107.5 \%$ were obtained with RSDs below $6.8 \%$ (Table $\mathrm{S} 2 \dagger$ ). Diluted human serum samples were selected as biological media to assess the practicality of the sensor for AA detection. Fig. S7† shows that the fluorescence intensity of the PDA NPs enhanced progressively as the AA concentration increased and a linear response to AA ranging from 0-20 $\mu \mathrm{M}$ was observed. In terms of the calibration curve of the serum, four serum samples with different concentrations of AA were prepared and measured, and satisfactory recoveries of between $\mathbf{9 5 . 6 \%}$ and $\mathbf{1 0 2 . 8 \%}$ were obtained with RSDs of around $5 \%$ (Table $\mathrm{S} 3 \dagger$ ). The results demonstrated that the developed strategy has potential practicability for $\mathrm{Hg}^{2+}$ and AA detection in real samples.

\section{Conclusions}

In summary, the rationale of a simple, rapid and sensitive nanosensor using fluorescent PDA NPs for the sequential detection of $\mathrm{Hg}^{2+}$ and $\mathrm{AA}$ has been demonstrated. The sensor was constructed mainly based on $\mathrm{H}^{2+}{ }^{2+}$-induced fluorescence quenching effect toward PDA NPs through an intense coordination interaction between $\mathrm{Hg}^{2+}$ and the groups on the surface of the PDA NPs, and AA-triggered induced fluorescence recovery of the PDA NPs via a redox reaction between $\mathrm{Hg}^{2+}$ and AA. By employing the fluorescent PDA NPs as signal readout molecules, the nanosensor exhibited high sensitivity and desirable selectivity. Moreover, the method showed potential applications for $\mathrm{Hg}^{2+}$ and AA detection, with satisfactory recoveries in real samples, which may provide new insight for applications in environmental analysis and biological detection diagnosis.

\section{Conflicts of interest}

There are no conflicts to declare.

\section{Acknowledgements}

We gratefully acknowledge the support from the NNSF of China (No. 21705068 and 21676131), the Fundamental Research Funds for Promotion Project of Jiangxi Science and Technology Normal University (2018BSQD024), and the Natural Science Foundation of Jiangxi Province (20143ACB20012 and 20192BAB213015).

\section{Notes and references}

1 S. A. El-Safty and M. A. Shenashen, TrAC, Trends Anal. Chem., 2012, 38, 98-115.

2 F. Zahir, S. J. Rizwi, S. K. Haq and R. H. Khan, Environ. Toxicol. Pharmacol., 2005, 20, 351-360.

3 K. A. Naidu, Nutr. J., 2003, 2, 7-16.

4 O. Arrigon and M. C. De Tullio, Biochim. Biophys. Acta, 2002, 1569, 1-9.

5 B. Halliwell, Annu. Rev. Nutr., 1996, 16, 33-50.

6 O. Blokhina, E. Virolainen and K. Fagerstedt, Ann. Bot., 2003, 91, 179-194.

7 N. Li, Y. Li, Y. Han, W. Pan, T. Zhang and B. Tang, Anal. Chem., 2014, 86, 3924-3930. 
8 L. K. Massey, M. Liebman and S. A. Kynast-Gales, J. Nutr., 2005, 135, 1673-1677.

9 S. G. Liu, D. Luo, L. Han, N. B. Li and H. Q. Luo, Microchim. Acta, 2019, 186, 156-163.

10 X. Jiang, H. Wang, H. Wang, R. Yuan and Y. Chai, Anal. Chem., 2016, 88, 9243-9250.

11 M. Zhang, K. Liu, L. Xiang, Y. Lin, L. Su and L. Mao, Anal. Chem., 2007, 79, 6559-6565.

12 Q. Li, J. Wang and Y. He, Sens. Actuators, B, 2016, 231, 64-69.

13 S. Zhao, Y. Huang and Y. M. Liu, J. Chromatogr. A, 2009, 1216, 6746-6751.

14 Y. S. Ho and P. C. Uden, J. Chromatogr. A, 1994, 688, 107-116.

15 Y. Wei, Z. Zhang, Y. Zhang and Y. Sun, Chromatographia, 2007, 65, 443-446.

16 X. Liu, Z. Wu, Q. Zhang, W. Zhao, C. Zong and H. Gai, Anal. Chem., 2016, 88, 2119-2124.

17 T. Wu, W. Hou, Z. Ma, M. Liu, X. Liu, Y. Zhang and S. Yao, Microchim. Acta, 2019, 186, 123-129.

18 W. Gu, X. Pei, Y. Cheng, C. Zhang, J. Zhang, Y. Yan, C. Ding and Y. Xian, ACS Sens., 2017, 2, 576-582.

19 X. Zhang, Z. Dai, S. Si, X. Zhang, W. Wu, H. Deng, F. Wang, X. Xiao and C. Jiang, Small, 2017, 13, 1603347-1603353.

20 H. M. Meng, X. B. Zhang, C. Yang, H. Kuai, G. J. Mao, L. Gong, W. Zhang, S. Feng and J. Chang, Anal. Chem., 2016, 88, 6057-6063.

21 H. Chen, Y. Liu, H. Li, Y. Zhang and S. Yao, Sens. Actuators, B, 2019, 281, 983-988.

22 W. Xu, J. Chen, S. Sun, Z. Tang, K. Jiang, L. Song, Y. Wang, C. Liu and H. Lin, Nanoscale, 2018, 10, 17834-17841.

23 J. Yu, W. Yang, S. Xing, J. Wang, H. Han and P. Zhang, Microchim. Acta, 2019, 186, 89-97.

24 H. Liu, W. Na, Z. Liu, X. Chen and X. Su, Biosens. Bioelectron., 2017, 92, 229-233.

25 H. B. Wang, Y. Li, H. Y. Bai and Y. M. Liu, Sens. Actuators, B, 2018, 259, 204-210.

26 A. N. Vijayan, Z. Liu, H. Zhao and P. Zhang, Anal. Chim. Acta, 2019, 1072, 75-80.

27 Y. Cen, J. Tang, X. J. Kong, S. Wu, J. Yuan, R. Q. Yu and X. Chu, Nanoscale, 2015, 7, 13951-13957.

28 D. Wang, C. Chen, X. Ke, N. Kang, Y. Shen, Y. Liu, X. Zhou, H. Wang, C. Chen and L. Ren, ACS Appl. Mater. Interfaces, 2015, 7, 3030-3040.

29 L. Li, C. Wang, K. Liu, Y. Wang, K. Liu and Y. Lin, Anal. Chem., 2015, 87, 3404-3411.

30 X. Zhu, T. Zhao, Z. Nie, Y. Liu and S. Yao, Anal. Chem., 2015, 87, 8524-8530.
31 L. L. Feng, Y. X. Wu, D. L. Zhang, X. X. Hu, J. Zhang, P. Wang, Z. L. Song, X. B. Zhang and W. Tan, Anal. Chem., 2017, 89, 4077-4084.

32 P. Zhao, K. He, Y. Han, Z. Zhang, M. Yu, H. Wang, Y. Huang, Z. Nie and S. Yao, Anal. Chem., 2015, 87, 9998-10005.

33 D. Yue, D. Zhao, J. Zhang, L. Zhang, K. Jiang, X. Zhang, Y. Cui, Y. Yang, B. Chen and G. Qian, Chem. Commun., 2017, 53, 11221-11224.

34 B. Chen, J. Ma, T. Yang, L. Chen, P. F. Gao and C. Z. Huang, Biosens. Bioelectron., 2017, 98, 36-40.

35 Y. Liu, K. Ai and L. Lu, Chem. Rev., 2014, 114, 5057-5115.

36 B. Xiong, Y. Chen, Y. Shu, B. Shen, H. N. Chan, Y. Chen, J. Zhou and H. Wu, Chem. Commun., 2014, 50, 13578-13580.

37 Q. Ci, J. Liu, X. Qin, L. Han, H. Li, H. Yu, K. L. Lim, C. W. Zhang, L. Li and W. Huang, ACS Appl. Mater. Interfaces, 2018, 10, 35760-35769.

38 H. Yin, K. Zhang, L. Wang, K. Zhou, J. Zeng, D. Gao, Z. Xia and Q. Fu, Nanoscale, 2018, 10, 18064-18073.

39 X. J. Kong, S. Wu, T. T. Chen, R. Q. Yu and X. Chu, Nanoscale, 2016, 8, 15604-15610.

40 T. Xiao, J. Sun, J. Zhao, S. Wang, G. Liu and X. Yang, ACS Appl. Mater. Interfaces, 2018, 10, 6560-6569.

41 Y. Liu, M. Yang, J. Li, W. Zhang and X. Jiang, Anal. Chem., 2019, 91, 6754-6760.

42 X. X. Wang, J. M. Liu, S. L. Jiang, L. Jiao, L. P. Lin and M. L. Cui, Sens. Actuators, B, 2013, 182, 205-210.

43 T. An, N. Lee, H. J. Cho, S. Kim, D. S. Shin and S. M. Lee, $R S C$ Adv., 2017, 7, 30582-30587.

44 S. Sharma, R. Uttam, A. S. Bharti and K. N. Uttam, Anal. Lett., 2019, 52, 1539-1557.

45 A. Sharma, J. Enderlein and M. Kumbhakar, J. Phys. Chem. Lett., 2017, 8, 5821-5826.

46 D. Su, M. Wang, Q. Liu, Z. Qu and X. Su, New J. Chem., 2018, 42, 17083-17090.

47 C. B. Ke, T. L. Lu and J. L. Chen, Microchim. Acta, 2019, 86, 376-383.

48 J. G. Egan, A. J. Hynes, H. M. Fruehwald, I. I. Ebralidze, S. D. King, R. A. M. Esfahani, F. Y. Naumkin, E. B. Easton and O. V. Zenkina, J. Mater. Chem. C, 2019, 7, 10187-10195.

49 R. Sanehi, R. M. Mehrotra and S. P. Mushran, J. Inorg. Nucl. Chem., 1975, 37, 1753-1755.

50 Q. Zou, X. Li, T. Xue, J. Zheng and Q. Su, Talanta, 2019, 195, 497-505.

51 N. O. Laschuk, I. I. Ebralidze, D. Spasyuk and O. V. Zenkina, Eur. J. Inorg. Chem., 2016, 3530-3535. 